\title{
Effect of novel $N$-arylurea- substituted 3-morpholino arecoline derivatives as muscarinic receptor 1 agonists in Alzheimer's dementia models
}

\author{
Y. C. Sunil Kumar, ${ }^{a}$ Manish Malviya, ${ }^{a}$ J. N. Narendra Sharath Chandra, ${ }^{a}$ C. V. Kavitha, \\ N. R. Thimmegowda, ${ }^{a}$ M. N. Subhash, ${ }^{b}$ and K. S. Rangappa ${ }^{a}$. \\ ${ }^{a}$ Department of Studies in Chemistry, University of Mysore, Manasagangotri, Mysore-570006, \\ India \\ ${ }^{b}$ Department of Neurochemistry, National Institute of Mental Health and Neurosciences, \\ Bangalore-560 029, India \\ E-mail: rangappaks@gmail.com,rangappaks@,chemistry.uni-mysore.ac.in
}

\begin{abstract}
The cholinergic hypothesis of Alzheimer's disease has spurred the development of numerous compounds aimed at increasing central cholinergic neurotransmission. Symptomatic treatment can be given by cholinomimetics with the pharmacological profile of muscarinic receptor 1 (M1 receptor) agonist and/or acetylcholineesterase (AChE) inhibitors. Novel bioactive six-membered $N$-arylurea- substituted 3-morpholino arecoline derivatives were synthesized by $N$-benzyl aminoethanol coupling with $\alpha$-bromoacetylpyridine followed by reduction and cyclization. Five of the derivatives showed high M1 receptor binding affinity in vitro and elicited beneficial effects in in vivo memory and learning models in rats.
\end{abstract}

Keywords: Alzheimer's diseases, M1 agonist, morpholino arecolines, displacement assay, rat brain

\section{Introduction}

Neurochemical examination of the brain material from patients having Alzheimer's disease (AD) has demonstrated the loss of the presynaptic marker enzyme, choline acetyltransferase, and the muscarinic receptors of the M2 subtype which are mainly responsible for causing deficits in central cholinergic transmission in Alzheimer's patients. ${ }^{1-3}$ The postsynaptic muscarinic receptors, which are primarily of the M1 subtype, seem to a large extent to survive the loss of cholinergic nerve endings. ${ }^{4}$ These findings have led to attempts at restoring cholinergic function by means of cholinomimetic drugs such as acetylcholinesterase (AChE) inhibitors and muscarinic agonists, the hypothesis being that enhancement of cholinergic neurotransmission 
would alleviate the symptoms of the diseases, and particularly the deficits in cognition and memory. ${ }^{4}$ Pharmacological investigation of muscarinic receptor subtypes using both functional and binding studies has identified three distinct muscarinic receptor subtypes, ${ }^{5}$ M1, M2 and M3. Identifying M1 selective muscarinic agonists which are capable of crossing the blood-brain barrier is the subject of active research for pharmacological application. ${ }^{6}$

Arecoline, an alkaloid obtained from the betel nut (Areca catechu), the fruit of a palm tree, has been used previously as a leading centrally active muscarinic agent. ${ }^{7}$ The lack of M1 selectivity and efficacy due to dose limiting side effects associated with M2 and M3 muscarinic receptor subtype stimulation have produced disappointing results. ${ }^{7}$ Replacement of the ester functionality of arecoline with either the 3-alkoxy-1,2,5 thiadiazole $^{8}$ or the 3-alkyl-1,2,4oxadiazole ${ }^{9}$ has produced very potent muscarinic agonists. However, the systematic removal of a heteroatom in the 3-methyl-1,2,4-oxadiazole, giving oxazoles or furans, caused a decrease in affinity for the agonist binding site. The two isomers, 2-methyl-1,2,4-oxadiazole and 5-methyl1,2,4-oxadiazole also had lower affinities for muscarinic receptors. ${ }^{9}$ No muscarinic M1 subtype selectivity has been reported for 2-(1-methyl-1,2,5,6-tetrahydropyridin-3-yl)morpholine. CFunctionalized morpholines are found in various naturally occurring products as well as in drugs. ${ }^{10}$ Since the compound 2-(1-methyl-1,2,5,6-tetrahydropyridin-3-yl)morpholine is a conformationally restricted arecoline analog, we were encouraged to pursue this compound.
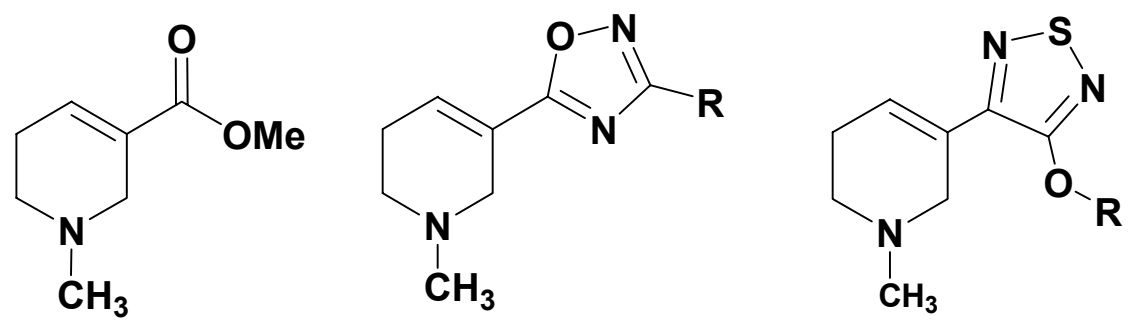

Arecoline

3-alkyl-1,2,4-oxadiazole

3-alkoxy-1,2,5-thiadiazole<smiles>[R]N1OC=NC1C1=CCCN(C)C1</smiles>

2-methyl-1,2,4-oxadiazole<smiles>[R]c1nc(C2=CCCN(C)C2)no1</smiles>

5-methyl-1,2,4-oxadiazole

Figure 1. Arecoline and arecoline derivatives.

In an earlier paper, we reported arecoline-thiazolidinones as a muscarinic receptor 1 agonist. ${ }^{11}$ Herein, we describe the synthesis of $N$-arylurea- substituted morpholino- arecolines 9(a-j), along with their in vitro muscarinic binding assay by using $\left[{ }^{3} \mathrm{H}\right]-\mathrm{QNB}$ with male wistar rat 
brain synaptosomal membrane and in vivo evaluation of memory and learning in male wistar rats, for the symptomatic treatment of Alzheimer's dementia.

\section{Chemistry}

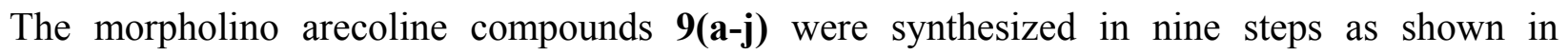
Scheme 1. Bromination of 3-acetylpyridine 1 with $\mathrm{Br}_{2} / \mathrm{HBr}$ in glacial acetic acid gave the $\mathrm{HBr}$ salt of bromoacetylpyridine, 2. This was converted into the amino-alcohol 3 by reaction with $N$ benzylaminoethanol in DMF in the presence of $\mathrm{K}_{2} \mathrm{CO}_{3}$. The keto group of compound 3 was reduced using $\mathrm{NaBH}_{4}$ in methanol to obtain the dihydroxy compound 4 . Treatment of 4 with $70 \% \mathrm{H}_{2} \mathrm{SO}_{4}$ under reflux conditions caused dehydration, to yield the cyclized product $\mathbf{5}$. The $N$ benzyl group of 5 was removed by refluxing in methanol in the presence of $10 \% \mathrm{Pd}-\mathrm{C}$ and ammonium formate, and the resulting free amine was treated with Boc-anhydride in THF in the presence of $\mathrm{K}_{2} \mathrm{CO}_{3}$ to yield the Boc-protected compound 6 . This was converted by reaction with methyl iodide in acetone into the corresponding methylamine hydro-iodide salt. This on treatment with sodium borohydride in methanol gave the reduced product 7 . Finally, the Boc group was removed using methanolic $\mathrm{HCl}$ to yield the free amine $\mathbf{8}$ as its $\mathrm{HCl}$ salt. The detailed procedure for the synthesis of compound $\mathbf{8}$ has been reported in our previous paper. ${ }^{12}$ This on reacting with the respective isocyanates gave the $N$-arylurea- substituted 3-morpholino-arecoline derivatives 9(a-j). ${ }^{1} \mathrm{H}$ NMR spectra of all compounds $9(\mathbf{a}-\mathbf{j})$ showed a multiplet at $\delta$ 9-7 due to aromatic protons and 5.7-5.8 due to the double bond of tetrahydropyridine. All the synthesized compounds were characterized by IR, ${ }^{1} \mathrm{H}$ NMR, mass spectroscopy, and CHN analysis.

\section{Results and Discussion}

A structure-activity relationship (SAR) can be drawn from the in vitro affinity assay for the synthesized $N$-arylurea- substituted 3-morpholino arecoline derivatives $\mathbf{9}(\mathbf{a}-\mathbf{j})$. Five of them showed greater affinity towards the M1 receptor (Table 1), in the order $9 \mathbf{9}>\mathbf{9 b}>\mathbf{9 e}>\mathbf{9 f}>\mathbf{9 j}$. The most potent compound among all tested derivatives is the one with the ethylbenzene group, $\mathbf{9 i}$ $\left(\mathrm{K}_{\mathrm{i}}=5 \mu \mathrm{M}\right)$, (Scheme 1 and Fig. 2).

Substitution of an electron-donating methoxy group, at the meta position (9b) of the aryl group attached to the nitrogen of urea, also showed good affinity towards the M1 receptor in vitro. However, when the same is introduced at an ortho- $(\mathbf{9 a})$ or a para- $(\mathbf{9 c})$ position it reduces the affinity of the compound towards the M1 receptor. A chlorine atom on the benzene ring, which acts as an electron- donating group by resonance, and electron withdrawing by the inductive effect, increases the affinity of the compound when present at a meta position (9e). However when it is present either at an ortho- (9d) or a para-position, (9f), it decreases the affinity of the compounds towards the M1 receptor. Introduction of a fluoro group at the paraposition $(\mathbf{9 g})$, or chloro groups ortho or para $(\mathbf{9 h})$ does not increase the affinity of the compound 
for the receptor. Compound $\mathbf{9 j}$ with an $\alpha$-methylbenzyl group substituted on the nitrogen of urea showed moderate activity towards the receptor in vitro.
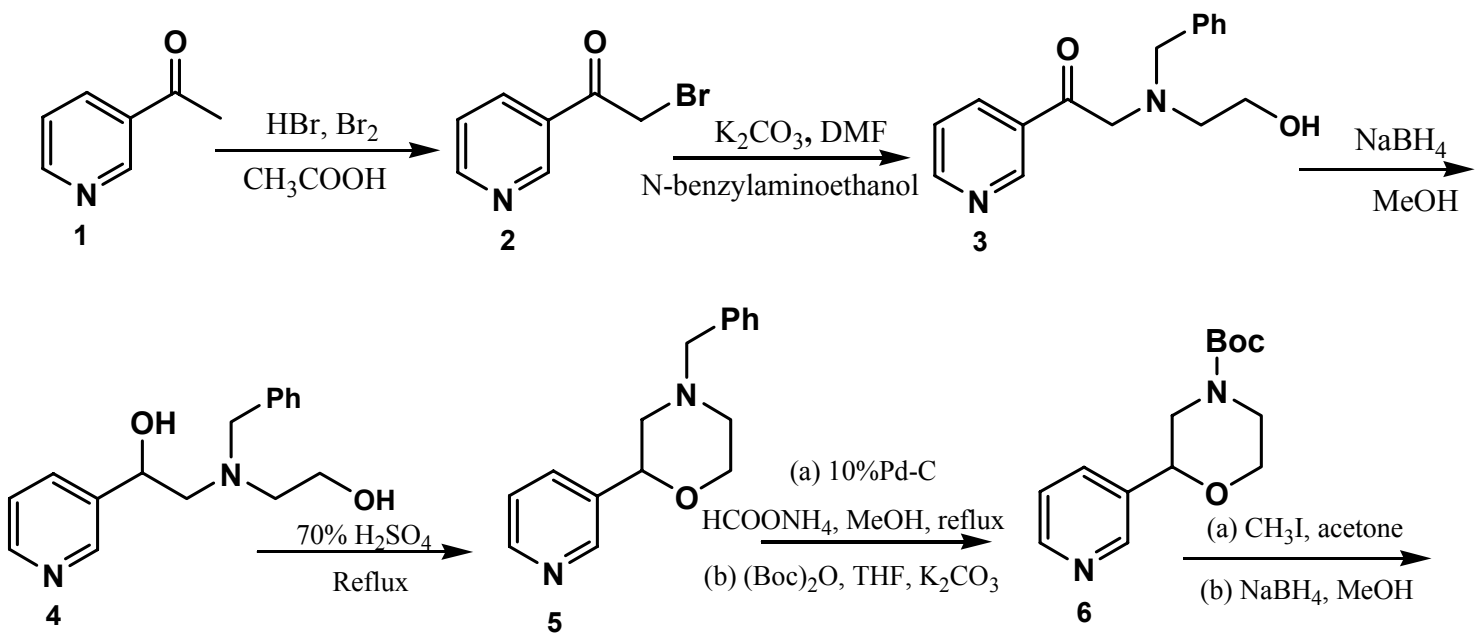<smiles>CN1CCC=C(C2CN(C(=O)OC(C)(C)C)CCO2)C1</smiles>

7<smiles>COc1cccc(SCc2cc(-c3cccc(OC)c3)ccc2OC)c1</smiles><smiles>CN1CCC=C(C2CNCCO2)C1</smiles>

8<smiles>[R]NC(=O)N1CCOC(C2=CCCN(C)C2)C1</smiles>

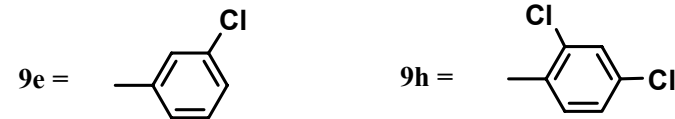<smiles>[18O]=Cc1ccc(Cl)cc1</smiles>

$9 \mathbf{i}=$<smiles>CC(C)c1ccccc1</smiles>

\section{Scheme 1}


Table 1. In vitro affinity and potency of $N$-arylurea- substituted 3-morpholino-arecoline derivatives $\mathbf{9}(\mathbf{a}-\mathbf{j})$ towards M1 receptor of male wistar rat cortex synaptosomal membrane

\begin{tabular}{ccc}
\hline Compounds & $\mathrm{Ki}(\mu \mathrm{M})$ & $\mathrm{IC}_{50}(\mu \mathrm{M})$ \\
\hline $\mathbf{9 a}$ & $38 \pm 4.36$ & $112 \pm 6.47$ \\
$\mathbf{9 b}$ & $12 \pm 2.08$ & $46 \pm 4.21$ \\
9c & $110 \pm 12.36$ & $512 \pm 19.23$ \\
9d & $89 \pm 4.92$ & $234 \pm 11.69$ \\
9e & $17 \pm 2.98$ & $61 \pm 11.87$ \\
9f & $26 \pm 3.22$ & $90 \pm 9.65$ \\
9g & $64 \pm 7.28$ & $169 \pm 15.12$ \\
9h & $40 \pm 5.36$ & $126 \pm 13.56$ \\
9i & $05 \pm 0.90$ & $21 \pm 8.56$ \\
9j & $31 \pm 5.36$ & $98 \pm 8.56$ \\
Arecoline & $86 \pm 8.39$ & $469 \pm 17.54$ \\
\hline
\end{tabular}

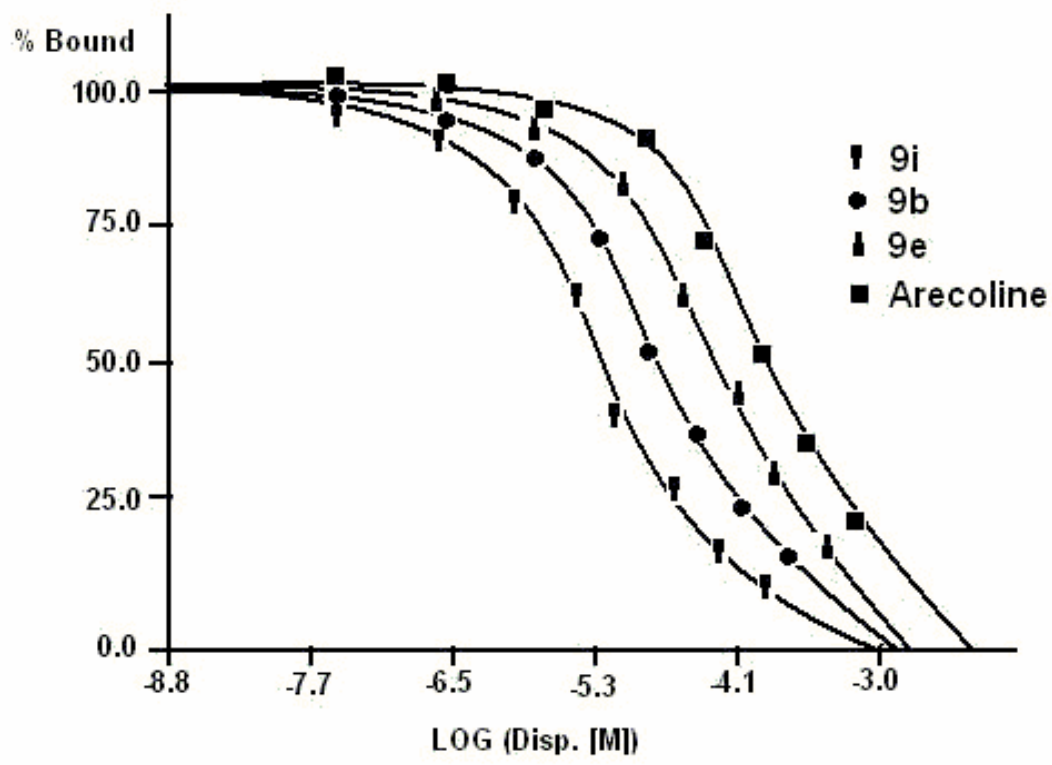

Figure 2. Displacement graph of three potent compounds $9 \mathbf{i}, \mathbf{9 b}$ and $9 \mathbf{e}$. The displacement studies were done with $0.2 \mathrm{n} M\left[{ }^{3} \mathrm{H}\right] \mathrm{QNB}$ and different concentration of $N$-arylurea- substituted 3-morpholino-arecolines $\mathbf{9}(\mathbf{a}-\mathbf{j})$. The mean values of $\%$ bound are plotted against the log of the displacer concentration. $\mathrm{IC}_{50}$ and $K i$ values are obtained from the Ligand-Drug program software. 
Table 2. Study of anti-amnesic effect of $N$-arylurea- substituted 3-morpholino-arecoline

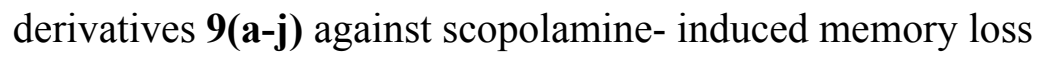

\begin{tabular}{|c|c|c|c|c|c|c|c|c|}
\hline \multirow[t]{2}{*}{$\begin{array}{l}\text { S1. } \\
\text { No. }\end{array}$} & \multirow[t]{2}{*}{$\begin{array}{c}\text { Experimental } \\
\text { groups }\end{array}$} & \multirow[t]{2}{*}{$\begin{array}{l}\text { Treatment } \\
\text { (dose) } \\
\text { mg/kg i.p. }\end{array}$} & \multicolumn{3}{|c|}{$\begin{array}{c}\text { Basal latency (sec.) of } \\
\text { rat to reach shock- free } \\
\text { zone (SFZ) }\end{array}$} & \multicolumn{2}{|c|}{ Memory parameters } & \multirow[t]{2}{*}{ t-test } \\
\hline & & & I & II & III & $\begin{array}{l}\text { Latency } \\
\text { (Seconds) }\end{array}$ & Mistakes & \\
\hline 1. & Control group* & $\begin{array}{l}\text { Saline } \\
(0.9 \%)\end{array}$ & 18 & 9 & 5 & 2 & $8 \pm 2.3$ & $\mathrm{t}_{10}=10.54$ \\
\hline 2. & $\begin{array}{l}\text { Scopolamine } \\
\text { treated group }\end{array}$ & 0.4 & 38 & 12 & 10 & 7 & $33 \pm 7.9$ & \\
\hline 3. & 9a* + Scop. & $0.1+0.4$ & 26 & 14 & 7 & 5 & $19 \pm 5.6$ & $\mathrm{t}_{10}=4.69$ \\
\hline 4. & 9b* + Scop. & $0.1+0.4$ & 20 & 10 & 7 & 3 & $10 \pm 3.0$ & $\mathrm{t}_{10}=9.32$ \\
\hline 5 & 9c + Scop. & $0.1+0.4$ & 38 & 14 & 10 & 7 & $32 \pm 8.4$ & $\mathrm{t}_{10}=0.26$ \\
\hline 6. & 9d + Scop. & $0.1+0.4$ & 34 & 14 & 9 & 6 & $30 \pm 6.6$ & $\mathrm{t}_{10}=0.93$ \\
\hline 7. & $9 \mathrm{e}^{*}+$ Scop. & $0.1+0.4$ & 21 & 11 & 6 & 4 & $11 \pm 2.5$ & $\mathrm{t}_{10}=9.18$ \\
\hline 8. & $\mathbf{9 f} *+$ Scop. & $0.1+0.4$ & 24 & 12 & 7 & 4 & $15 \pm 3.9$ & $\mathrm{t}_{10}=6.87$ \\
\hline 9. & $\mathbf{9} \mathbf{g} * *+$ Scop. & $0.1+0.4$ & 30 & 13 & 8 & 5 & $22 \pm 4.8$ & $\mathrm{t}_{10}=3.92$ \\
\hline 10. & $\mathbf{9 h} *+$ Scop & $0.1+0.4$ & 28 & 13 & 8 & 5 & $20 \pm 4.5$ & $\mathrm{t}_{10}=4.74$ \\
\hline 11. & $9 \mathbf{i}^{*}+$ Scop & $0.1+0.4$ & 20 & 10 & 6 & 3 & $10 \pm 2.6$ & $\mathrm{t}_{10}=9.54$ \\
\hline 12. & $\mathbf{9 j} *+$ Scop. & $0.1+0.4$ & 25 & 13 & 7 & 4 & $18 \pm 6.3$ & $\mathrm{t}_{10}=4.76$ \\
\hline
\end{tabular}

Results expressed as mean $( \pm \mathrm{SEM}), n=8(\mathrm{Scop}=$ Scopolamine $0.4 \mathrm{mg} / \mathrm{kg}$, i.p $) . * \mathrm{P}=<0.001$, $* * \mathrm{P}=<0.002$, Degrees of freedom for each parameter is 14 .

The aforesaid in vitro M1 receptor binding studies formed a basis for extending the correlation further to in vivo pharmacological studies, to ascertain the applicability of the

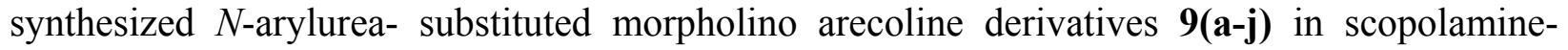
induced dementia models (male wistar rats) using memory- and learning- experiments (passive avoidance tasks). In accordance with the degree of affinity and potency of the compounds $\mathbf{9}(\mathbf{a}-\mathbf{j})$, in vitro binding experiments elicited almost the anticipated level of pharmacological actions in reversing scopolamine-induced dementia in vitro (Table 2).

Three of the synthesized morpholino- arecoline derivatives, 9i, 9b and 9e, reversed scopolamine-induced dementia by making rats make fewer mistakes (number of mistakes made, 10, 10 and 11, respectively) when compared with the number of mistakes made by the control rats (8 mistakes) and the scopolamine-treated group (33 mistakes). Compounds 9f, 9j and 9a also significantly reversed the scopolamine- induced memory loss. Compounds 9c and 9d were among the least potent compound among the all tested derivatives (Figure 3). 


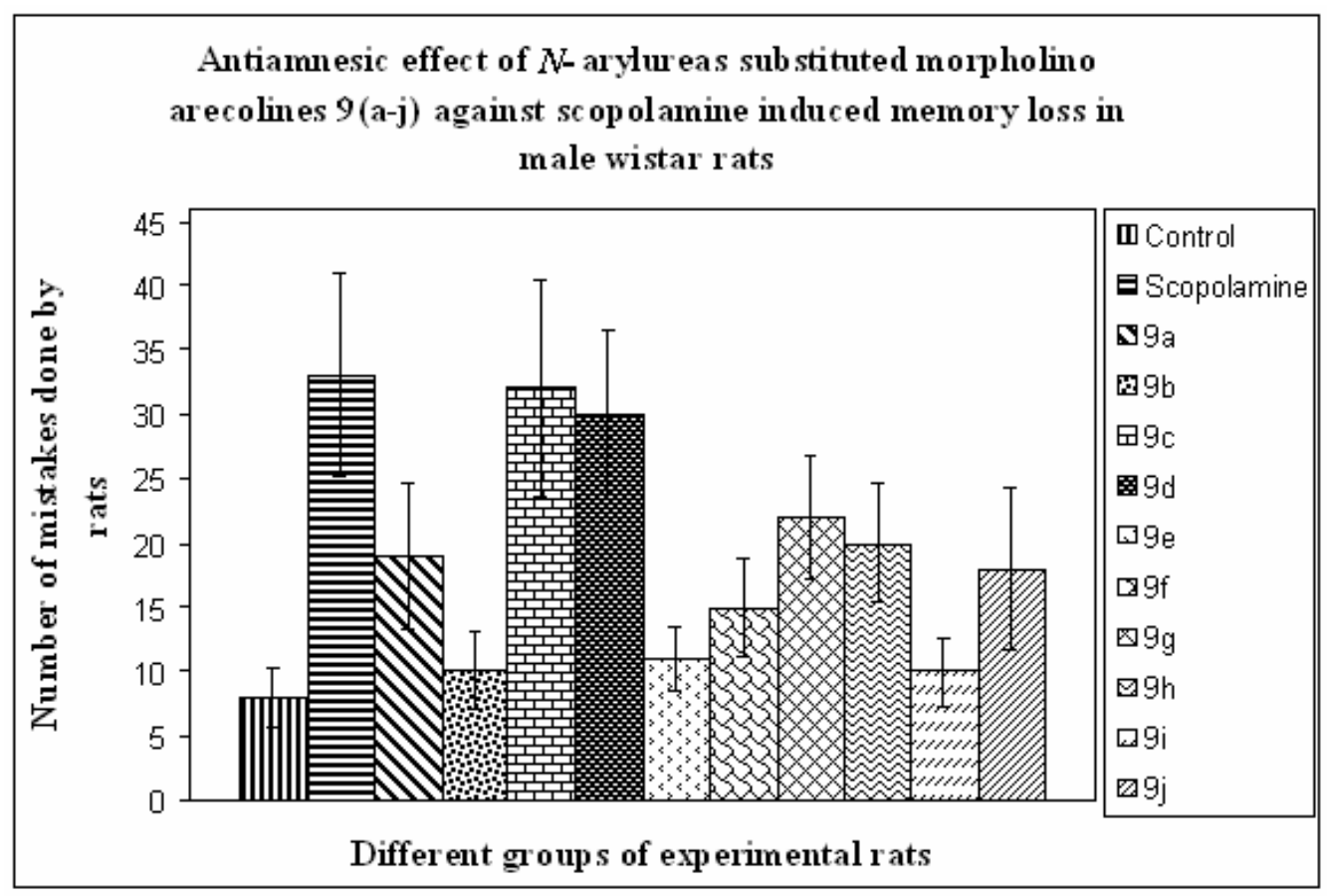

Figure 3. Anti-dementia activity of $N$-arylurea- substituted 3-morpholino-arecolines $\mathbf{9}(\mathbf{a}-\mathbf{j})$. Mean ( \pm SEM).

\section{Conclusions}

In light of these findings, in vitro competitive M1 receptor displacement assay using male wistar rat-brain synaptosomal membrane and in vivo pharmacological experiments for the synthesized compounds 9(a-j) testify to the reversal of scopolamine- induced memory loss and learning impairment in male wistar rats to ascertain their applicability in dementia. The derivatives with methoxy group/s at the meta position of the phenyl group (9b) showed considerably high affinity and potency for the M1 receptor in vitro, and useful anti-dementia activity in the in vivo model tested, but when the same is present at an ortho- or para-position, it decreases the affinity for the receptor. The compound $9 \mathbf{i}$ which has an ethylbenzene unit is found to have the highest affinity for the M1 receptor when compared with the other tested compounds. Compound $\mathbf{9 e}$, which has a $\mathrm{Cl}$ group at a meta position showed good affinity when compared with ortho- $\mathrm{Cl}$ in $\mathbf{9 d}$ or para$\mathrm{Cl}$ in $9 \mathbf{f}$.

From the above results, we can conclude that if the $\mathrm{Cl}$ or OMe substitution is present at a meta position, the affinity of the compounds increases, whereas when it is present at an ortho or para position, the affinity of compounds decreases. This may be due to the resonance effect where electrons from $\mathrm{OMe}$ or $\mathrm{Cl}$ from ortho- or para- positions delocalize towards the nitrogen of urea via the benzene ring, which is not observed when they are present at a meta position. 
These $N$-aryl morpholino arecoline derivatives $\mathbf{9 ( a - j )}$ showed no visible cholinergic toxicity (salivation, defecation, etc.) at the dose tested.

\section{Experimental Section}

General Procedures. Infrared (IR) spectra were recorded using Nujol on JASCO-FTIR, 4100 series. ${ }^{1} \mathrm{H}$ NMR spectra were recorded on Shimadzu AMX 400-Bruker, 400MHz spectrometer using $\mathrm{CDCl}_{3}$ as solvent and TMS as internal standard (chemical shifts in $\delta$ ppm). Spin multiplets are given as s (singlet), $\mathrm{d}$ (doublet), $\mathrm{t}$ (triplet) and $\mathrm{m}$ (multiplet). Mass and purity were recorded on a LC-MSD-Trap-XCT. Elemental (CHN) analyses were obtained on Vario EL III Elementar. Silica gel column chromatography was performed using Merck 7734 silica gel (60-120 mesh) and Merck made TLC plates. All chemicals and reagents were obtained from Aldrich (USA), Spectrochem Pvt. Ltd (India), or Rankem Pvt. Ltd. (India) and were used without further purification.

General procedure for the synthesis $N$-Aryl ureas substituted 3-morpholino-Arecoline derivatives $9(\mathbf{a}-\mathbf{j})$

The intermediate compound 2-(1-methyl-1,2,5,6-tetrahydropyridin-3-yl)morpholine (8, Scheme 1) was synthesised in eight steps as described previously. ${ }^{12}$ To a solution of compound 8 $(1 \mathrm{eq})$ in dichloromethane, triethylamine $(5 \mathrm{eq})$ was added and cooled to $0^{0} \mathrm{C}$. The respective isocyanate (1 eq) was added in the cold and stirred at room temperature for 4-5 $\mathrm{hr}$ (completion of reaction confirmed by TLC). Water was added and the product extracted thrice using dichloromethane. The combined organic layer was washed with brine and dried over anhydrous sodium sulphate. The dichloromethane was evaporated under reduced pressure and the crude product was purified on a silica gel (60-120 mesh) column. The compounds $\mathbf{9 ( a - j ) ~ w e r e ~ e l u t e d ~ a t ~}$ $8 \%$ to $10 \%$ methanol in chloroform.

\section{2-(1,2,5,6-Tetrahydro-1-methylpyridin-3-yl)- $N$-(2-methoxyphenyl)morpholine 4-carboxa-} mide (9a). Obtained by reaction of compound 8 (0.2 g, $0.00078 \mathrm{~mol})$ with 2-methoxy-phenyl isocyanate $(0.117 \mathrm{~g}, 0.00078 \mathrm{~mol})$ and triethylamine $(0.394 \mathrm{~g}, 0.0039 \mathrm{~mol})$ in dichloromethane (3 ml). Yield, 82\%; IR: 3201 (-NH-),1623 (-CO-N-), 1675 (-RC=CH-). ${ }^{1} \mathrm{H}$ NMR: $\delta$ 8.21(s, 1H), 7.13-7.10 (m, 1H), 6.88-6.86 (m, 2H), 6.784-6.763 (d, 1H, $J=8.4$ Hz), 5.76 (bs, 1H, -C=CH-), $3.85-3.74(\mathrm{~m}, 3 \mathrm{H}), 3.73(\mathrm{~s}, 3 \mathrm{H}), 3.52-3.48(\mathrm{~m}, 1 \mathrm{H}), 3.31-3.26(\mathrm{~m}, 3 \mathrm{H}), 2.80-2.76(\mathrm{~m}, 2 \mathrm{H}), 2.25-$ $2.48(\mathrm{~m}, 2 \mathrm{H}), 2.21(\mathrm{~s}, 3 \mathrm{H}), 2.02(\mathrm{~m}, 2 \mathrm{H})$. MS (m/z): 332..51 $\left(\mathrm{M}^{+}\right)$. Anal. Calcd for $\mathrm{C}_{18} \mathrm{H}_{25} \mathrm{~N}_{3} \mathrm{O}_{3}$ : C, 65.23; H, 7.60; N, 12.68. Found: C, 65.48; H, 7.83; N, 12.41\%.

\section{2-(1,2,5,6-Tetrahydro-1-methylpyridin-3yl)- $\mathrm{N}$-(3-methoxyphenyl)morpholine 4-carboxa-} mide (9b). Obtained by reaction of compound $8(0.2 \mathrm{~g}, 0.00078 \mathrm{~mol})$ with 3 -methoxy-phenyl isocyanate $(0.117 \mathrm{~g}, 0.00078 \mathrm{~mol})$ and triethylamine $(0.394 \mathrm{~g}, 0.0039 \mathrm{~mol})$ in dichloromethane (3 ml). Yield: 83\%; IR (Nujol, $\left.\mathrm{cm}^{-1}\right)$ : 3206 (-NH-), 1628 (-CO-N-) 1676 (RC=CH-). ${ }^{1} \mathrm{H}$ NMR $\left(\mathrm{CDCl}_{3}\right): \delta 8.02(\mathrm{~s}, 1 \mathrm{H}), 7.35-7.21(\mathrm{~m}, 1 \mathrm{H}), 6.90-6.86(\mathrm{~m}, 2 \mathrm{H}), 6.67-6.65(\mathrm{dd}, 1 \mathrm{H}, J=1.6$ and $1.6 \mathrm{~Hz}), 5.74$ (bs, $1 \mathrm{H},-\mathrm{C}=\mathrm{CH}-), 3.84-3.73(\mathrm{~m}, 3 \mathrm{H}), 3.70(\mathrm{~s}, 3 \mathrm{H}), 3.53-3.49(\mathrm{~m}, 1 \mathrm{H}), 3.33-3.28$ 
(m, 3H), 2.81-2.76 (m, 2H), 2.52-2.47 (m, 2H), $2.23(\mathrm{~s}, 3 \mathrm{H}), 2.01(\mathrm{~m}, 2 \mathrm{H}) . \mathrm{MS}(\mathrm{m} / \mathrm{z}): 332.8$ $\left(\mathrm{M}^{+}\right)$; Anal. Calcd for $\mathrm{C}_{18} \mathrm{H}_{25} \mathrm{~N}_{3} \mathrm{O}_{3}$ : C, 65.23; H, 7.60; N, 12.68. Found: C, 64.98; H, 7.73; N, $12.50 \%$.

\section{2-(1,2,5,6-Tetrahydro-1-methylpyridin-3yl)- $N$-(4-methoxyphenyl)morpholine-4-carboxa-}

mide (9c). Obtained by reaction of compound $8(0.2 \mathrm{~g}, 0.00078 \mathrm{~mol})$ with 4-methoxy- phenyl isocyanate $(0.117 \mathrm{~g}, 0.00078 \mathrm{~mol})$ and triethylamine $(0.394 \mathrm{~g}, 0.0039 \mathrm{~mol})$ in dichloromethane (3 ml). Yield: 86\%; IR (Nujol, $\left.\mathrm{cm}^{-1}\right)$ : 3204(-NH-), 1628 (-CO-N-) 1678 (-RC=CH-). ${ }^{1} \mathrm{H}$ NMR $\left(\mathrm{CDCl}_{3}\right): \delta 8.22(\mathrm{~s}, 1 \mathrm{H}), 7.315-7.296(\mathrm{~d}, 2 \mathrm{H}, J=7.6 \mathrm{~Hz}), 6.863-6.842(\mathrm{~d}, 2 \mathrm{H}, J=8.4 \mathrm{~Hz}), 5.74$ (bs, $1 \mathrm{H},-\mathrm{C}=\mathrm{CH}-), 3.85-3.74(\mathrm{~m}, 3 \mathrm{H}), 3.74(\mathrm{~s}, 3 \mathrm{H}), 3.52-3.47(\mathrm{~m}, 1 \mathrm{H}), 3.33-3.28(\mathrm{~m}, 3 \mathrm{H}), 2.84-$ $2.79(\mathrm{~m}, 2 \mathrm{H}), 2.53-2.48(\mathrm{~m}, 2 \mathrm{H}), 2.21(\mathrm{~s}, 3 \mathrm{H}), 2.00(\mathrm{~m}, 2 \mathrm{H}) . \mathrm{MS}(\mathrm{m} / \mathrm{z}): 332.41\left(\mathrm{M}^{+}\right)$; Anal. Calcd for $\mathrm{C}_{18} \mathrm{H}_{25} \mathrm{~N}_{3} \mathrm{O}_{3}$ : C, 65.23; H,7.60; N, 12.68. Found: C, 64.89; H, 7.86; N, $12.51 \%$.

$\mathrm{N}$-(2-Chlorophenyl)-2-(1,2,5,6-tetrahydro-1-methylpyridin-3-yl)morpholine-4-carboxamide (9d). Obtained by reaction of compound $8(0.2 \mathrm{~g}, 0.00078 \mathrm{~mol})$ with 2-chloro-phenyl isocyanate $(0.120 \mathrm{~g}, 0.00078 \mathrm{~mol})$ and triethylamine $(0.394 \mathrm{~g}, 0.0039 \mathrm{~mol})$ in dichloromethane $(3 \mathrm{ml})$.Yield: 88 \%; IR: 3221 (-NH-), 1644 (-CO-N-), 1678 (-RC=CH-). ${ }^{1} \mathrm{H}$ NMR $\left(\mathrm{CDCl}_{3}\right): \delta 8.22$ (s, 1H), 7.472-7.453 (d, 1H, $J=7.6 \mathrm{~Hz}), 7.25-7.20$ (m, 3H), 5.73 (bs, $1 \mathrm{H},-\mathrm{C}=\mathrm{CH}-), 3.80-3.75(\mathrm{~m}, 3 \mathrm{H})$, 3.53-3.50 (m, 1H), 3.30-3.23 (m, 3H), 2.80-2.72 (m, 2H), 2.54-2.46 (m, 2H), $2.26(\mathrm{~s}, 3 \mathrm{H}), 2.05$ $(\mathrm{m}, 2 \mathrm{H})$. MS $(\mathrm{m} / \mathrm{z}): 336.90\left(\mathrm{M}^{+}\right)$. Anal. Calcd for $\mathrm{C}_{17} \mathrm{H}_{22} \mathrm{ClN}_{3} \mathrm{O}_{2}: \mathrm{C}, 60.80 ; \mathrm{H}, 6.60 ; \mathrm{N}, 12.51$. Found: C, 60.83; H, 6.75; N, 12.53\%.

$\mathrm{N}$-(3-Chlorophenyl)-2-(1,2,5,6-tetrahydro-1-methylpyridin-3-yl)morpholine-4-carboxamide (9e). Obtained by reaction of $8(0.2 \mathrm{~g}, 0.00078 \mathrm{~mol})$ with 3-chlorophenyl isocyanate $(0.120 \mathrm{~g}$, $0.00078 \mathrm{~mol})$ and triethylamine $(0.394 \mathrm{~g}, 0.0039 \mathrm{~mol})$ in dichloromethane $(3 \mathrm{ml})$. Yield: $92 \%$; IR: 3224(-NH-), 1643(-CO-N-) 1677 (-RC=CH-). ${ }^{1} \mathrm{H}$ NMR $\left(\mathrm{CDCl}_{3}\right): \delta 8.03(\mathrm{~s}, 1 \mathrm{H}), 7.395-7.390$ $(\mathrm{d}, 1 \mathrm{H}, J=2.06 \mathrm{~Hz}), 7.26-7.20(\mathrm{~m}, 2 \mathrm{H}), 7.132-7.116(\mathrm{~d}, 1 \mathrm{H}, J=7.2 \mathrm{~Hz}), 5.73$ (bs, 1H, $-\mathrm{C}=\mathrm{CH}-)$, 3.84-3.78 (m, 3H), 3.50-3.48 (m, 1H), 3.33-3.27 (m, 3H), 2.80-2.75 (m, 2H), 2.53-2.48 (m, 2H), $2.24(\mathrm{~s}, 3 \mathrm{H}), 2.04(\mathrm{~m}, 2 \mathrm{H})$. MS (m/z): $336.90\left(\mathrm{M}^{+}\right)$; Anal. Calcd for $\mathrm{C}_{17} \mathrm{H}_{22} \mathrm{ClN}_{3} \mathrm{O}_{2}: \mathrm{C}, 60.80 ; \mathrm{H}$, $6.60 ;$ N, 12.51. Found: C, 60.88; H, 6.75; N, 12.83\%.

$\mathrm{N}$-(4-Chlorophenyl)-2-(1,2,5,6-tetrahydro-1-methylpyridin-3-yl)morpholine-4-carboxamide (9f). Obtained by reaction of $8(0.2 \mathrm{~g}, 0.00078 \mathrm{~mol})$ with 4-chlorophenyl isocyanate $(0.120 \mathrm{~g}$, $0.00078 \mathrm{~mol})$ and triethylamine $(0.394 \mathrm{~g}, 0.0039 \mathrm{~mol})$ in dichloromethane $(3 \mathrm{ml})$. Yield: $92 \%$; IR: 3221 (-NH-), 1646 (-CO-N-), $1669(-\mathrm{RC}=\mathrm{CH}-) \mathrm{cm}^{-1}$; ${ }^{1} \mathrm{H}$ NMR $\left(\mathrm{CDCl}_{3}\right)$ : $\delta 8.22(\mathrm{~s}, 1 \mathrm{H})$, 7.351-7.331 (d, 2H, $J=8.4 \mathrm{~Hz}), 7.234-7.215$ (d, 2H, $J=7.6 \mathrm{~Hz}), 5.75$ (bs, 1H, -C=CH-), 3.84$3.79(\mathrm{~m}, 3 \mathrm{H}), 3.53-3.48(\mathrm{~m}, 1 \mathrm{H}), 3.33-3.28(\mathrm{~m}, 3 \mathrm{H}), 2.81-2.75(\mathrm{~m}, 2 \mathrm{H}), 2.55-2.48(\mathrm{~m}, 2 \mathrm{H}), 2.28$ (s, 3H), $2.05(\mathrm{~m}, 2 \mathrm{H})$. MS (m/z): $336.90\left(\mathrm{M}^{+}\right)$; Anal. Calcd for $\mathrm{C}_{17} \mathrm{H}_{22} \mathrm{ClN}_{3} \mathrm{O}_{2}$ : C, 60.80; H, 6.60; N, 12.51. Found: C, 60.85; H, 6.78; N, 12.55\%.

$\mathrm{N}$-(4-Fluorophenyl)-2-(1,2,5,6-tetrahydro-1-methylpyridin-3-yl)morpholine-4-carboxamide $(9 \mathrm{~g})$. Obtained by reaction of $8(0.2 \mathrm{~g}, 0.00078 \mathrm{~mol})$ with 4-fluorophenyl isocyanate $(0.107 \mathrm{~g}$, $0.00078 \mathrm{~mol})$ and triethylamine $(0.394 \mathrm{~g}, 0.0039 \mathrm{~mol})$ in dichloromethane $(3 \mathrm{ml})$. Yield: $90 \%$; IR: 3232 (-NH-), 1638 (-CO-N-), $1675 \mathrm{~cm}^{-1}$ (-RC=CH-). ${ }^{1} \mathrm{H}$ NMR $\delta 8.20$ (s, 1H), 7.416-7.404 (d, $2 \mathrm{H}, J=8.8 \mathrm{~Hz}$ ), 7.102-7.120 (d, 2H, $J=7.2 \mathrm{~Hz}), 5.73$ (bs, 1H, -C=CH-), 3.85-3.75 (m, 3H), 
3.53-3.48 (m, 1H), 3.33-3.27 (m, 3H), 2.81-2.76 (m, 2H), 2.54-2.49 (m, 2H), $2.24(\mathrm{~s}, 3 \mathrm{H}), 2.04$ $(\mathrm{m}, 2 \mathrm{H})$. MS $(\mathrm{m} / \mathrm{z}): 320.38\left(\mathrm{M}^{+}\right)$. Anal. Calcd for $\mathrm{C}_{17} \mathrm{H}_{22} \mathrm{FN}_{3} \mathrm{O}_{2}$ : C, 63.93; H, 6.94; N, 13.16 . Found: C, 64.20; H, 6.83; N, 13.34\%.

$\mathrm{N}$-(2,4-dichlorophenyl)-2-(1,2,5,6-tetrahydro-1-methylpyridin-3-yl)morpholine-4-carboxamide (9h). Obtained by reaction of compound 8 ( $0.2 \mathrm{~g}, 0.00078 \mathrm{~mol})$ with 2,4-dichloro- phenyl isocyanate $(0.148 \mathrm{~g}, 0.00078 \mathrm{~mol})$ and triethylamine $(0.394 \mathrm{~g}, 0.0039 \mathrm{~mol})$ in dichloromethane (3 ml). Yield: 94\%; IR: 3243 (-NH-), 1633 (-CO-N-), $1676(-\mathrm{RC}=\mathrm{CH}-) .{ }^{1} \mathrm{H}$ NMR $\left(\mathrm{CDCl}_{3}\right): \delta$ $8.20(\mathrm{~s}, 1 \mathrm{H}), 7.596-7.591(\mathrm{~d}, 1 \mathrm{H}, J=2.0 \mathrm{~Hz}), 7.368-7.347(\mathrm{~d}, 1 \mathrm{H}, J=8.4 \mathrm{~Hz}), 7.512-7.492(\mathrm{~d}$, $1 \mathrm{H}, J=8.0 \mathrm{~Hz}), 5.70$ (bs, $1 \mathrm{H},-\mathrm{C}=\mathrm{CH}-), 3.80-3.76(\mathrm{~m}, 3 \mathrm{H}), 3.54-3.50(\mathrm{~m}, 1 \mathrm{H}), 3.30-3.28(\mathrm{~m}$, $3 \mathrm{H}), 2.81-2.75(\mathrm{~m}, 2 \mathrm{H}), 2.50-2.47(\mathrm{~m}, 2 \mathrm{H}), 2.26(\mathrm{~s}, 3 \mathrm{H}), 2.06-2.04(\mathrm{~m}, 2 \mathrm{H}) . \mathrm{MS}(\mathrm{m} / \mathrm{z}): 370.86$ $\left(\mathrm{M}^{+}\right)$; Anal. Calcd for $\mathrm{C}_{17} \mathrm{H}_{21} \mathrm{Cl}_{2} \mathrm{~N}_{3} \mathrm{O}_{2}$ : C, 55.14; H, 5.72; N, 11.35. Found: C, 55.34; H, 5.84; N, $11.30 \%$.

2-(1,2,5,6-Tetrahydro-1-methylpyridin-3yl)- $N$-phenethylmorpholine-4- carboxamide (9i).

Obtained by reaction of compound $8(0.2 \mathrm{~g}, 0.00078 \mathrm{~mol}), 1$-(2-isocyanatoethyl) benzene $(0.115 \mathrm{~g}, 0.00078 \mathrm{~mol})$ and triethylamine $(0.394 \mathrm{~g}, 0.0039 \mathrm{~mol})$ in dichloromethane $(3 \mathrm{ml})$. Yield: 87\%; IR (Nujol): 3211 (-NH-), 1638 (-CO-N-), $1676(-\mathrm{RC}=\mathrm{CH}-) .{ }^{1} \mathrm{H}$ NMR $\left(\mathrm{CDCl}_{3}\right): \delta$ $8.22(\mathrm{~s}, 1 \mathrm{H}), 7.30(\mathrm{~s}, 5 \mathrm{H}), 5.76(\mathrm{~b}, 1 \mathrm{H},-\mathrm{C}=\mathrm{CH}-), 3.85-3.75(\mathrm{~m}, 3 \mathrm{H}), 3.50-3.45(\mathrm{~m}, 1 \mathrm{H}), 3.31-$ $3.20(\mathrm{~m}, 3 \mathrm{H}), 2.79-2.76(\mathrm{~m}, 2 \mathrm{H}), 2.52-2.43(\mathrm{~m}, 2 \mathrm{H}), 2.23(\mathrm{~s}, 3 \mathrm{H}), 2.10-2.09(\mathrm{~m}, 2 \mathrm{H}), 2.02(\mathrm{~m}$, 4H). MS (m/z): $330.54\left(\mathrm{M}^{+}\right)$. Anal. Calcd for $\mathrm{C}_{19} \mathrm{H}_{27} \mathrm{~N}_{3} \mathrm{O}_{2}: \mathrm{C}, 69.27 ; \mathrm{H}, 8.26 ; \mathrm{N}, 12.76$. Found: $\mathrm{C}$, $68.98 ; \mathrm{H}, 8.38 ; \mathrm{N}, 12.69 \%$.

\section{2-(1,2,5,6-Tetrahydro-1-methylpyridin-3yl)- $N$-(1-phenethyl)morpholine-4- carboxamide}

(9j). Obtained by reaction of compound 8 (0.2 g, $0.00078 \mathrm{~mol})$ with 1-(1-isocyanatoethyl) benzene $(0.115 \mathrm{~g}, 0.00078 \mathrm{~mol})$ and triethylamine $(0.394 \mathrm{~g}, 0.0039 \mathrm{~mol})$ in dichloromethane (3 ml). Yield: 89\%; IR (Nujol, $\left.\mathrm{cm}^{-1}\right)$ : 3212 (-NH-), 1640(-CO-N-), 1674 (-RC=CH-). ${ }^{1} \mathrm{H}$ NMR $\left(\mathrm{CDCl}_{3}\right): \delta 8.21(\mathrm{~s}, 1 \mathrm{H}), 7.31-7.06(\mathrm{~m}, 2 \mathrm{H}), 6.95-6.90(\mathrm{~m}, 3 \mathrm{H}), 5.76(\mathrm{~b}, 1 \mathrm{H},-\mathrm{C}=\mathrm{CH}-), 3.85-3.76$ $(\mathrm{m}, 3 \mathrm{H}), 3.53-3.49(\mathrm{~m}, 1 \mathrm{H}), 3.32-3.28(\mathrm{~m}, 3 \mathrm{H}), 2.79-2.78(\mathrm{~m}, 2 \mathrm{H}), 2.53-2.48(\mathrm{~m}, 2 \mathrm{H}), 2.20(\mathrm{~s}$, $3 \mathrm{H}), 2.04(\mathrm{~m}, 2 \mathrm{H}), 1.24(\mathrm{~m}, 3 \mathrm{H})$. MS (m/z): $330.50\left(\mathrm{M}^{+}\right)$. Anal. Calcd for $\mathrm{C}_{19} \mathrm{H}_{27} \mathrm{~N}_{3} \mathrm{O}_{2}: \mathrm{C}, 69.27$; H, 8.26; N, 12.76. Found: C, 69.30; H, 8.38; N, 12.65\%.

\section{Biology}

\section{Displacement study}

The competitive inhibition study was done using various synthesized $N$-arylurea- substituted 3morpholino arecoline derivatives $\mathbf{9}(\mathbf{a}-\mathbf{j})$ to find their affinity towards cortical M1 receptor. Male wistar rat brain cortex was taken out and used for synaptosomal membrane preparation. A crude membrane pellet was obtained from brain tissue, and homogenized in 20 volumes of Tris- $\mathrm{HCl}$ buffer $(50 \mathrm{mmol} / \mathrm{L}, \mathrm{pH} 7.4$ ) containing $0.32 \mathrm{~mol} / \mathrm{L}$ sucrose, following the procedure described by Creese and Snyder. ${ }^{13}$ The tissue homogenate was centrifuged at a speed of $1,000 \mathrm{~g}$ for 10 minutes at $4^{0} \mathrm{C}$, to remove cellular debris. The supernatant obtained was centrifuged at $32,000 \mathrm{~g}$ for 20 minutes at $4^{0} \mathrm{C}$. The pellet obtained was re-suspended in $50 \mathrm{mmol} / \mathrm{L}$ phosphate assay buffer 
( $\mathrm{pH}$ 7.4) containing $1 \mathrm{mmol} \mathrm{MgCl}_{2}$. The protein concentration was estimated by the method described by Lowry et al. ${ }^{14}$

The affinity of various compounds towards M1 receptor was estimated by using [ $\left.{ }^{3} \mathrm{H}\right] \mathrm{QNB}$ (0.2 nM, specific activity 48Ci/mmol, Amersham, Little Chalfont, Bucks, UK) essentially following the procedure described by Hyttel et al. ${ }^{15}$ and Yamamura and Snyder $^{16}$ with slight modification. In brief, an aliquot of synaptosomal membrane proteins (50 $\mu \mathrm{g})$ was incubated with different concentrations of compounds (0.1-200 $\mu \mathrm{M})$ as a displacer and $\left[{ }^{3} \mathrm{H}\right] \mathrm{QNB}(0.2 \mathrm{nM})$ and the reaction volume was made up to $200 \mu \mathrm{l}$ with assay buffer in 96 well plates and incubated for $2 \mathrm{hrs}$ at $37{ }^{\circ} \mathrm{C}$. The reaction for all displacement assays was stopped by adding ice-cold assay buffer and the reaction mixtures were rapidly filtered through GF/B filters under vacuum. The filters were transferred to vials and $5 \mathrm{ml}$ of scintillation fluid was added and allowed to equilibrate overnight. Radioactivity was measured in a liquid scintillation counter (Tris-Carb 2100 TR, Packard, US) at $65 \%$ efficiency.

The data from displacement were analysed and $\mathrm{IC}_{50}$ and $\mathrm{Ki}$ values are obtained from LigandDrug programme. ${ }^{17}$ The mean values of $\%$ bound are plotted against the $\log$ of displacer concentration.

\section{Anti-amnesic activity}

This was carried out for the synthesized $N$-aryl-morpholino- arecoline derivatives $\mathbf{9 ( a - j ) ~ a g a i n s t ~}$ scopolamine- induced memory loss using passive avoidance step down task paradigm in male wistar rats weighing 200-250gm $(n=8)$ according to the method described by Sharma and Kulkarni. $^{18,19}$

\section{Acute toxicity}

Rats ( 8 per group) which had fasted $16 \mathrm{hr}$, were treated orally with various doses of the compounds and observed for 1 week after treatment; deaths were recorded daily. None of the rats died within one week after administration under the test dose.

\section{Dose-response curve}

Different doses $(0.05-0.2 \mathrm{mg} / \mathrm{kg})$ of the derivatives were selected to find the optimum dose (found to be $0.1 \mathrm{mg} / \mathrm{kg}$ ) for in vivo studies.

\section{Data analysis}

The data from the displacement assay were analyzed using "LIGAND-DRUG" software programme $^{17}$ to obtain the IC50 and Ki values (both are expressed in $\mu \mathrm{Mol}$ ). All the data are expressed as mean $\pm \mathrm{SD}$. The statistical analysis was done by using student's t-test. Differences were considered to be significant at $\mathrm{P}<0.05$. All analysis was performed with the "JandelScientific-Sigma stat" software, version 2.0 for Windows. 


\section{Acknowledgements}

The authors are grateful to the Department of Science and Technology (DST), New Delhi, for financial support under the project SR/SO/HS-58/2003. The CHN, IR and other data were obtained from the instruments granted under DST-FIST and UGC-SAP (phase I) programs and are gratefully acknowledged. One of the authors (Mansih Malviya) is grateful to the Indian Council of Medical Research (ICMR) for providing Senior Research Fellowship.

\section{References}

1. Perry, E. K. Br. Med. Bull. 1986, 42, 63.

2. Sims, N. R.; Bowen, D. M.; Smith, C. C. T.; Neary, D.; Thomas, D. J.; Davison, A. N. J. Neurochem. 1983, 40, 503.

3. Perry, E. Br. Psychiat. 1988, 152, 737.

4. Narang, P. K.; Cuter, N. R. Prog. Neuro-Psychopharmacol. Biol. Psychiat. 1986, 10, 519.

5. Mutschler, E.; Moser, U.; Wess, J.; Lambrecht, G. In Progress in Pharmacology and Clinical Pharmacology; Gustav Fischer: Stuttgart, New York, 1989; Vol. 7/1, p. 13.

6. Sauerberg, P.; Olesen, H. P.; Nielsen, S.; Treppendahl, S.; Sheardown, J. M.; Honore, T.; Mitch, H. C.; Ward, S. J.; Pike, J. A.; Bymaster, P. F.; Sawyer, D. B.; Shannon, E. H. J. Med. Chem. 1992, 32, 2274.

7. Christie, J. E.; Shering, A.; Ferguson, J.; Glen, A. I. M. Br. J. Psychiat. 1981, 138, 46.

8. Sauerberg, P.; Preben H. Olesen.; Susanne Nielsen, et al. J. Med. Chem. 1992, 35, 2274.

9. Sauerberg, P.; Kindtler, J. W.; Nielsen, L.; Sheardown M. J.; Honore, T. J. Med. Chem. 1991, 34, 687.

10. Wijtmass, R.; Vink, K. S. M.; Schoemaker, E. H.; VanDelft, F. L.; Blaauw, R. H.; Rutjes, P. J. T. Synthesis 2004, 5, 641.

11. Chandra, J. N.; Malviya, M.; Sadashiva, C. T.; Subhash, M. N.; Rangappa, K. S. Neurochem Int. 2008, 52, 376.

12. Sunil Kumar, Y. C.; Sadashiva, M. P.; Rangappa, K. S. Tetrahedron Letters 2007, 48, 4565.

13. Creese, I.; Snyder, S. H. Eur J Pharmaco. 1978, 49, 201.

14. Lowry, O. H.; Rosebrough, N. J.; Farr, A.L.; Randall, R. J. J Biol Chem. 1951, 193, 265.

15. Hyttel, J.; Nielsen, J. B.; Nowak, G. J Neural Transm Gen Sect. 1992, 89, 61.

16. Yamamura, H.I.; Snyder, S.H. Proc Natl Acad Sci U S A 1974, 71, 1725.

17. McPherson, G. A. Comput Programs Biomed. 1983, 17,107.

18. Sharma, A.C.; Kulkarni, S. K. Methods Find Exp Clin Pharmacol. 1990, 12, 175.

19. Sharma, A.C.; Kulkarni, S. K. Methods Find Exp Clin Pharmacol. 1991, 13, 155. 